\title{
Influence of cytochrome P450 2D6 polymorphism on hippocampal white matter and treatment response in schizophrenia
}

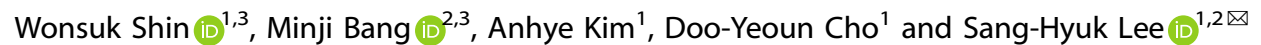

\begin{abstract}
Cytochrome P450 2D6 (CYP2D6) is expressed at high levels in the brain and plays a considerable role in the biotransformation and neurotransmission of dopamine. This raises the question of whether CYP2D6 variations and its impact on the brain can confer susceptibility to schizophrenia. We investigated the possible links among the CYP2D6 genotype, white matter (WM) integrity of the hippocampus, and the treatment response to antipsychotic drugs in Korean patients with schizophrenia $(n=106)$. Brain magnetic resonance imaging and genotyping for CYP2D6 were conducted at baseline. The severity of clinical symptoms and the treatment response were assessed using the Positive and Negative Syndrome Scale (PANSS). After genotyping, 43 participants were classified as intermediate metabolizers (IM), and the remainder $(n=63)$ were classified as extensive metabolizers (EM). IM participants showed significantly higher fractional anisotropy (FA) values in the right hippocampus compared to EM participants. Radial diffusivity (RD) values were significantly lower in the overlapping region of the right hippocampus in the IM group than in the EM group. After 4 weeks of antipsychotic treatment, the EM group showed more improvements in positive symptoms than the IM group. FAs and RDs in the CYP2D6-associated hippocampal WM region were significantly correlated with a reduction in the positive symptom subscale of the PANSS. Greater improvements in positive symptoms were negatively associated with FAs, and positively associated with RDs in the right hippocampal region. The findings suggest that CYP26D-associated hippocampal WM alterations could be a possible endophenotype for schizophrenia that accounts for individual differences in clinical features and treatment responses.
\end{abstract}

npj Schizophrenia (2021)7:5; https://doi.org/10.1038/s41537-020-00134-z

\section{INTRODUCTION}

Cytochrome P450 2D6 (CYP2D6), a member of the cytochrome P450 mixed-function oxidase system, is one of the most popular enzymes involved in the metabolism of numerous drugs used in clinical practice. The CYP2D6 gene is highly polymorphic with allelic variants causing a large variability in enzymatic activity. Based on what alleles are carried, individuals are classified as poor $(P M)$, intermediate (IM), extensive (EM), and ultrarapid (UM) metabolizers ${ }^{1}$. Recent evidence shows that CYP2D6 is expressed at high levels in neuronal cells of the brain $^{2,3}$, and plays a considerable role in the biotransformation and neurotransmission of central dopamine ${ }^{4}$. This raises the question of whether individual variations in CYP2D6 can confer susceptibility to schizophrenia ${ }^{5}$.

CYP2D6 in the brain is colocated with the dopamine transporter on membranes of dopaminergic neurons ${ }^{6}$. CYP2D6 and the dopamine transporter are functionally similar because they share a common substrate- $\left[{ }^{3} \mathrm{H}\right] \mathrm{GBR}-12935$ - which labels the dopamine transporter complex ${ }^{7-9}$. CYP2D6 also has strong activity for the conversion of dopamine from tyramine that exists in the brain $^{10}$, suggesting its intertwining effects on the central dopaminergic system. Previous studies revealed that CYP2D6 activity is associated with conditions, in which dopamine signaling plays a substantial role in pathophysiology, including Parkinson's disease $^{11}$, smoking behavior ${ }^{12}$, sexual dysfunction ${ }^{13}$, and suicide ${ }^{14}$. In patients with schizophrenia, although some genetic studies produced negative results ${ }^{15-17}$, LLerena and colleagues reported that the frequency of PMs was significantly lower in patients than in healthy controls ${ }^{18}$. However, the relationship between CYP2D6 and schizophrenia is not conclusive in that the exact role of CYP2D6 in the brain, and dopaminergic system remains poorly understood.

The central expression of CYP2D6 has been found in several brain regions, particularly in the cornu ammonis 1-3 of the hippocampus $^{2,3}$. Hippocampal hyperactivity, which leads to the increased dopamine neuron firing and dopamine hyperresponsiveness, is one of the core mechanisms underlying the pathogenesis of schizophrenia ${ }^{19,20}$. Central CYP2D6 may have an additive effect on alterations in the dopamine concentration in the hippocampus by being involved in dopamine transmission and metabolism at the synapse. In this case, given that perturbations in dopamine signaling are associated with direct changes in white matter (WM) myelination ${ }^{8,21,22}$, it could be assumed that individual variations in CYP2D6 activity in patients with schizophrenia are further reflected in measures of WM integrity. Moreover, inter-patient differences in treatment response to antipsychotic drugs (APDs) could be affected by local cerebral drug metabolism by CYP2D6; although, its level in the brain is very low compared to that in the liver ${ }^{6,12}$. Since the current treatment of schizophrenia relies on APDs that block mesolimbic postsynaptic dopamine transmission ${ }^{23}$, CYP2D6 variability and its associated WM changes may be related to the treatment response of APDs in patients with schizophrenia.

\footnotetext{
${ }^{1}$ Department of Clinical Pharmacology and Therapeutics, CHA Bundang Medical Center, CHA University School of Medicine, Seongnam, Republic of Korea. ${ }^{2}$ Department of Psychiatry, CHA Bundang Medical Center, CHA University School of Medicine, Seongnam, Republic of Korea. ${ }^{3}$ These authors contributed equally: Wonsuk Shin, Minji Bang. email: drshlee@cha.ac.kr
} 
Ethnicity differences are particularly important in understanding the impact of CYP2D6 polymorphism on schizophrenia ${ }^{24}$. About $6-10 \%$ of Caucasians are reported as PM, and over $25 \%$ of Ethiopians are reported as $\mathrm{UM}^{25}$. However, in the Republic of Korea, the frequencies of PM and UM are $0.25 \%$ and $1.25 \%$, respectively ${ }^{26-28}$. Most of the CYP2D6 activity is expected in EM (64.25\%) and IM (34.25\%). CYP2D6*5 is the most common nonfunctional allele (6.13\%), and CYP2D6*10 is the most common allele for decreased function (45.00\%). The allele frequency of CYP2D6 varies among racial/ethnic groups; in particular, EM and IM alleles are most frequent in the Republic of Korea. Hence, exploring the clinical differences between EM and IM in the Korean population is of great significance.

We investigated the possible links among the CYP2D6 genotype, WM integrity of the hippocampus, and the treatment response to APDs in Korean patients with schizophrenia. We hypothesized that (1) hippocampal WM integrity, measured using diffusion tensor imaging (DTI), would differ between IM and EM patients with schizophrenia; (2) IM and EM patients would show different treatment response to APDs; and (3) CYP2D6-associated WM changes would be associated with treatment response to APDs.

\section{RESULTS}

Genotyping, sociodemographic, and clinical characteristics of the study participants

The genotype distributions of CYP2D6*5 and CYP2D6*10 in the entire group of participants with schizophrenia were in accordance with the Hardy-Weinberg equilibrium (CYP2D6*5, $-/-: n$ =93, -/del: $n=13$, del/del: $n=0, \quad x^{2}=0.45, \quad p=0.500$; CYP2D6*10, CC: $n=24, C T: n=39, T: n=30$, single allele: $n=$ $\left.13, x^{2}=2.31, p=0.129\right)$. Forty-three participants were categorized as IMs (activity score [AS] 0.5: $n=6$; AS 1.0: $n=37$ ), and the remaining 63 participants as EMs (AS 1.5: $n=39$; AS 2.0: $n=24$ ). Among the 65 participants who were followed-up for the treatment response after 4 weeks, the genotype distributions of CYP2D6*5 and CYP2D6* 10 were also in accordance with the Hardy-Weinberg equilibrium (CYP2D6*5, -/-: $n=58,-/$ del: $n=$ 7, del/del: $n=0, \chi^{2}=0.21, p=0.646 ;$ CYP2D6*10, CC: $n=15$, CT: $n$ $=25$, Tा: $n=18$, single allele: $n=7, x^{2}=1.07, p=0.302$ ). Twenty five participants belonged to the IM group (AS 0.5: $n=4$; AS 1.0: $n$ $=21$ ), and the others $(n=40)$ to the EM group (AS 1.5: $n=25$; AS 2.0: $n=15$ ). The frequency of IM and EM participants in this study was substantially the same as that reported in a previous study on CYP2D6 genotypes in the Korean population ${ }^{26}$.

Table 1 summarizes the sociodemographic and clinical characteristics of all study participants at baseline. The mean AS was nearly twofold higher in the EM group than in the IM group. There were no significant differences in other baseline characteristics between the IM and EM groups. Eighty five out of 106 participants $(80.2 \%)$ were recent-onset patients who had developed psychotic symptoms within 5 years. Eighty six participants (81.1\%) were APD-naive and the remainder were free of APDs at least for 6 months at the time of enrollment. Neuroimaging data were acquired within 2 weeks after the initiation of APD treatment, if participants were not cooperative because of exacerbated psychotic symptoms (mean duration of APD treatment before magnetic resonance imaging [MRI] scan: $4.4 \pm 4.2$ days).

\section{Baseline comparison of hippocampal WM connectivity between the IM and EM groups}

For neuroimaging analysis, hippocampal WM regions were defined as regions of interest (ROls) based on the Johns Hopkins University (JHU) DTI-based probabilistic tractography atlas (Fig. 1) ${ }^{29}$. Fractional anisotropy (FA) values were higher in the right hippocampus in the IM group than in the EM group (Fig. 2a, b). Radial diffusivity
Table 1. Sociodemographic and clinical characteristics of all subjects at baseline scan.

\begin{tabular}{|c|c|c|c|c|}
\hline & $\operatorname{IM}(n=43)$ & $\mathrm{EM}(n=63)$ & Statistics & $p$ Value \\
\hline Sex ( $n$, male/female) & $14 / 29$ & $21 / 42$ & $x^{2}=0.007$ & 0.934 \\
\hline Age (years, mean $\pm S D$ ) & $37.9 \pm 13.5$ & $35.5 \pm 10.9$ & $t=0.980$ & $0.330^{d}$ \\
\hline $\mathrm{ICV}(\mathrm{ml}$, mean $\pm \mathrm{SD})$ & $1453 \pm 160$ & $1465 \pm 148$ & $t=-0.421$ & 0.675 \\
\hline $\begin{array}{l}\text { Duration of illness } \\
\text { (months, mean } \pm \text { SD) }\end{array}$ & $47.3 \pm 80.5$ & $32.7 \pm 60.9$ & $t=1.06$ & 0.290 \\
\hline $\begin{array}{l}\text { Duration of untreated } \\
\text { psychosis (months, } \\
\text { mean } \pm \text { SD) }\end{array}$ & $13.0 \pm 32.0$ & $15.8 \pm 31.1$ & $t=-0.440$ & 0.661 \\
\hline $\begin{array}{l}\text { Duration of APD before } \\
\text { scan (days, mean } \pm \text { SD) }\end{array}$ & $4.8 \pm 4.9$ & $4.3 \pm 4.4$ & $t=0.815$ & $0.418^{\mathrm{d}}$ \\
\hline $\begin{array}{l}\text { APD dose at scan } \\
(\mathrm{mg} / \text { day, mean } \pm \mathrm{SD})^{\mathrm{a}}\end{array}$ & $542.3 \pm 281.4$ & $528.9 \pm 227.6$ & $t=0.269$ & 0.789 \\
\hline Maintenance APD & & & $x^{2}=4.243$ & 0.515 \\
\hline Paliperidone $(n)$ & 16 & 27 & & \\
\hline Risperidone (n) & 11 & 15 & & \\
\hline Amisulpride $(n)$ & 9 & 16 & & \\
\hline Olanzapine $(n)$ & 4 & 3 & & \\
\hline Clozapine $(n)$ & 2 & 0 & & \\
\hline Aripiprazole $(n)$ & 1 & 2 & & \\
\hline \multicolumn{5}{|l|}{$\begin{array}{l}\text { Other psychotropic } \\
\text { medication }\end{array}$} \\
\hline Mood stabilizer $(n)^{\mathrm{b}}$ & 4 & 3 & $x^{2}=0.854$ & 0.355 \\
\hline Antidepressant $(n)^{c}$ & 5 & 4 & $x^{2}=0.916$ & 0.338 \\
\hline $\begin{array}{l}\text { 2D6 activity score } \\
\text { (mean } \pm \text { SD) }\end{array}$ & $0.93 \pm 0.18$ & $1.69 \pm 0.24$ & $t=-18.628$ & $<0.001$ \\
\hline \multicolumn{5}{|l|}{ PANSS (mean \pm SD) } \\
\hline Positive symptom & $29.3 \pm 6.2$ & $29.9 \pm 8.2$ & $t=-0.381$ & 0.704 \\
\hline Negative symptom & $28.3 \pm 7.6$ & $25.4 \pm 9.7$ & $t=1.607$ & 0.111 \\
\hline $\begin{array}{l}\text { General } \\
\text { psychopathology }\end{array}$ & $60.1 \pm 14.6$ & $54.9 \pm 14.8$ & $t=1.805$ & 0.074 \\
\hline
\end{tabular}

IM intermediate metabolizer, $E M$ extensive metabolizer, $S D$ standard deviation, ICV intracranial volume, APD antipsychotic drug, PANSS Positive and Negative Syndrome Scale.

${ }^{\text {a } A P D}$ doses were converted to the equivalent of chlorpromazine.

${ }^{\mathrm{b}}$ All subjects receiving mood stabilizer were taking divalproex sodium.

'Antidepressants administered by subjects were escitalopram $(n=4)$, sertraline $(n=1)$, paroxetine $(n=3)$, and duloxetine $(n=1)$.

'Levene's test for equality of variances indicated that the variances did not assumed to be equal between the two groups.

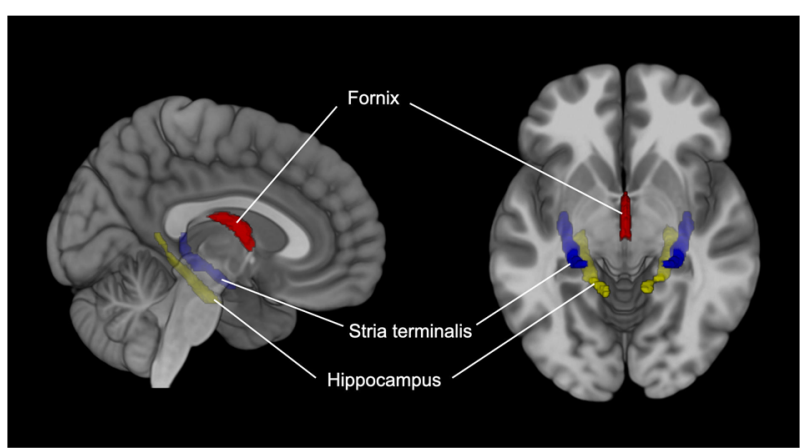

Fig. 1 White matter regions of interest. Hippocampal WM regions (hippocampal cingulum, fornix, and stria terminalis) were chosen as ROlsbased on the JHU DTI-based probabilistic tractography atlas.

(RD) values were significantly lower in the overlapping region of the hippocampus in the IM group as compared to the EM group (Fig. 2c, d). The result was not changed after controlling for sex, age, intracranial volume (ICV), duration of illness, and APD dose at scan as covariates. No significant differences were observed for axial (AD) and mean (MD) diffusivity between the two groups. 
(a)

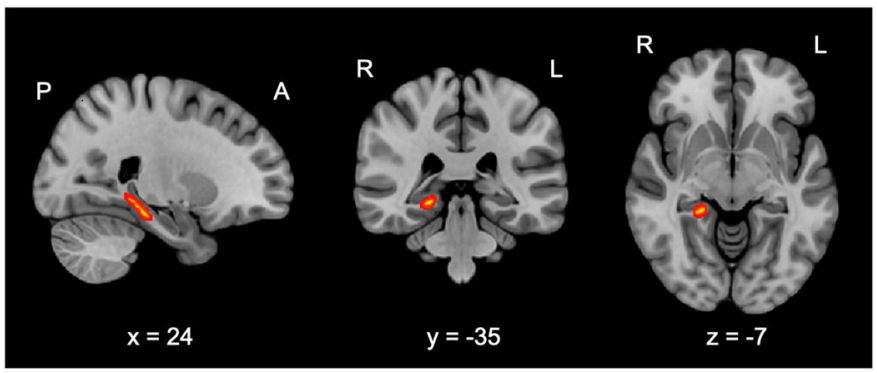

(c)

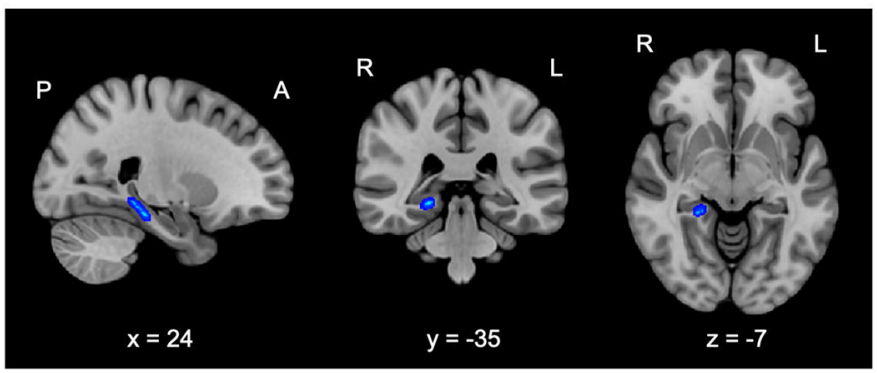

(b)

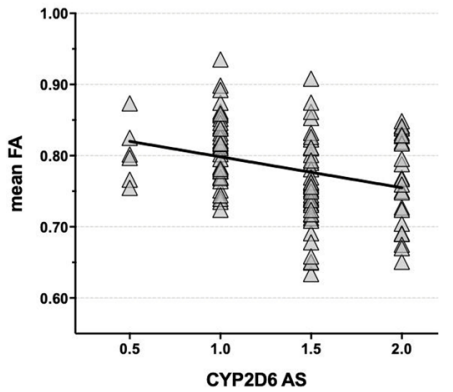

(d)

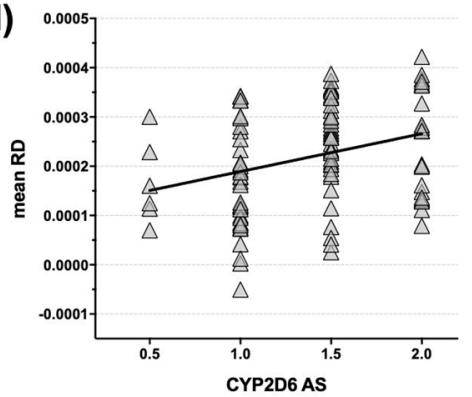

Fig. 2 Comparison of hippocampal white matter connectivity between IM and EM patients with schizophrenia. The IM group showed significantly increased FAs (a) and decreased RDs (c) compared to the EM group in the right hippocampal white matter region (TFCE-corrected $p$ < 0.05). The mean FAs (b) and RDs (d) in this region were significantly correlated with the activity score of CYP2D6 in participants with schizophrenia. IM intermediate metabolizer, EM extensive metabolizer, FA fractional anisotropy, RD radial diffusivity, TFCE threshold-free cluster enhancement.

\section{Relationships among CYP2D6 activity, hippocampal WM connectivity, and the treatment response}

Baseline characteristics between the IM and EM groups of 4-week follow-up participants were not significantly different except for the severity of general psychopathology (Table 2). Both groups were significantly improved across all symptom domains after 4 weeks of APD treatment (IM: paired- $t>11.651, p<0.001$; EM: paired- $t>11.957, p<0.001)$. EM patients showed a trend of lesssevere clinical symptoms than IM patients at the 4-week follow-up. When the percentages of reduction in the Positive and Negative Syndrome Scale (PANSS) ${ }^{30}$ subscale scores were compared, the EM group showed significantly greater improvements in positive symptoms compared to the IM group.

As shown in Fig. 3, voxel-wise correlation analysis in the CYP2D6-associated WM region of the right hippocampus revealed that FAs and RDs in this region were significantly associated with the percentages of reduction in positive symptoms. The percentages of reduction were negatively correlated with FAs (Fig. 3a, b) and positively correlated with RDs (Fig. 3c, d) in the right hippocampal region.

\section{DISCUSSION}

This study examined the genetic influence of CYP2D6 polymorphism on hippocampal WM connectivity and treatment response in patients with schizophrenia. We demonstrated that IM patients had significantly higher $F A$ and lower $R D$ values in the right hippocampal WM region compared to EM patients. In the entire group of patients with schizophrenia, the CYP2D6 ASs were associated with the FA and RD values of the right hippocampal WM region-negatively with the $F A$ and positively with the RD values. After 4 weeks of APD treatment, the EM group showed a significantly higher percentage of reduction in the severity of positive symptoms compared to the IM group. In accordance with aforementioned findings, higher FA and lower RD values in the CYP2D6 activity-associated WM cluster were significantly correlated with the less-improved percentages of reduction in the PANSS positive symptom subscale scores. The present results suggest that CYP2D6 polymorphism may affect structural alterations in WM connectivity of the brain underlying the pathogenesis and clinical manifestation of schizophrenia.

A lack of neuroimaging studies had investigated the impact of CYP2D6 polymorphism on the brain. A previous study with heathy individuals of central European origin showed that lower CYP2D6 activity was associated with higher levels in resting brain perfusion in the thalamus and hippocampus ${ }^{31}$. Elevated regional cerebral blood flow in the hippocampus provides supporting evidence for hippocampal hyperactivity in patients with schizophrenia ${ }^{32-34}$. Excessive hippocampal activity, which is mediated by the dysfunction of hippocampal gamma-aminobutyric acid interneurons $^{35}$, causes a overdrive of the mesolimbic dopamine system that leads to the development of schizophrenia ${ }^{36,37}$. Such neuronal stimulation induces adaptive myelination, resulting in an increase in myelin thickness and integrity of the downward WM pathways $^{38}$. Hence, we carefully assume that our finding of higher hippocampal WM connectivity in IMs with schizophrenia may reflect that the low activity of CYP2D6 is one contributing factor to hippocampal hyperactivity and dopamine dysregulation.

After 4 weeks of APD treatment, participants with schizophrenia who had extensive CYP2D6 activity showed a better improvement in positive symptoms than those with intermediate activity. From the pharmacological point of view, the response to APDs is correlated with their plasma concentration in most cases $^{39-41}$. In this study, the most frequently administered APDs in the follow-up group were paliperidone and amisulpride, which are mainly excreted by the kidney and are not vastly influenced by cytochrome P450 (refs. ${ }^{42,43}$ ). Since there was no significant between-group difference in maintenance APD doses, the exposure to APDs might not differ between the IM and EM groups. Therefore, our finding of different treatment response according to CYP2D6 activity may be owing to the ripple effect of CYP2D6 on central dopamine signaling, not the local cerebral metabolism of APDs. 
Table 2. Sociodemographic and clinical characteristics of the 4-week follow-up group.

\begin{tabular}{|c|c|c|c|c|}
\hline & IM $(n=25)$ & $\mathrm{EM}(n=40)$ & Statistics & $p$ Value \\
\hline Sex ( $n$, male/female) & 9/16 & $12 / 28$ & $x^{2}=0.253$ & 0.615 \\
\hline Age (years, mean $\pm S D$ ) & $40.1 \pm 13.3$ & $34.7 \pm 11.8$ & $t=1.711$ & 0.092 \\
\hline $\mathrm{ICV}(\mathrm{ml}$, mean $\pm \mathrm{SD})$ & $1458 \pm 167$ & $1468 \pm 154$ & $t=-0.239$ & 0.812 \\
\hline $\begin{array}{l}\text { Duration of illness } \\
\text { (months, mean } \pm \text { SD) }\end{array}$ & $47.6 \pm 85.2$ & $26.6 \pm 54.5$ & $t=1.100$ & $0.279^{e}$ \\
\hline $\begin{array}{l}\text { Duration of untreated } \\
\text { psychosis } \\
\text { (months, mean } \pm S D \text { ) }\end{array}$ & $12.3 \pm 24.0$ & $15.5 \pm 31.2$ & $t=-0.431$ & 0.668 \\
\hline $\begin{array}{l}\text { Duration of APD before scan } \\
\text { (days, mean } \pm \text { SD) }\end{array}$ & $5.3 \pm 5.1$ & $3.7 \pm 3.3$ & $t=1.341$ & $0.188^{e}$ \\
\hline $\begin{array}{l}\text { APD dose at scan (mg/day, } \\
\text { mean } \pm S D)^{a}\end{array}$ & $654.5 \pm 293.5$ & $528.7 \pm 214.2$ & $t=1.993$ & 0.051 \\
\hline $\begin{array}{l}\text { APD dose after } 4 \text { weeks } \\
(\mathrm{mg} / \mathrm{day}, \text { mean } \pm \mathrm{SD})^{\mathrm{b}}\end{array}$ & $789.6 \pm 276.3$ & $754.5 \pm 273.2$ & $t=0.501$ & 0.618 \\
\hline Maintenance APD & & & $x^{2}=5.020$ & 0.285 \\
\hline Paliperidone $(n)$ & 11 & 21 & & \\
\hline Risperidone $(n)$ & 2 & 6 & & \\
\hline Amisulpride $(n)$ & 7 & 11 & & \\
\hline Olanzapine $(n)$ & 3 & 2 & & \\
\hline Clozapine $(n)$ & 2 & 0 & & \\
\hline Aripiprazole $(n)$ & 0 & 0 & & \\
\hline \multicolumn{5}{|l|}{ Other psychotropic medication } \\
\hline Mood stabilizer $(n)^{c}$ & 4 & 7 & $x^{2}=0.025$ & 0.875 \\
\hline Antidepressant $(n)^{d}$ & 2 & 5 & $x^{2}=0.324$ & 0.569 \\
\hline $\begin{array}{l}\text { 2D6 activity score } \\
(\text { mean } \pm \text { SD) }\end{array}$ & $0.92 \pm 0.19$ & $1.69 \pm 0.25$ & $t=-14.246$ & $<0.001$ \\
\hline \multicolumn{5}{|l|}{ Baseline PANSS (mean \pm SD) } \\
\hline Positive symptom & $31.1 \pm 4.8$ & $31.2 \pm 8.7$ & $t=-0.042$ & $0.967^{f}$ \\
\hline Negative symptom & $30.6 \pm 6.8$ & $26.5 \pm 9.9$ & $t=1.815$ & 0.074 \\
\hline General psychopathology & $65.0 \pm 12.1$ & $56.8 \pm 15.1$ & $t=2.303$ & 0.025 \\
\hline \multicolumn{5}{|c|}{ PANSS after 4 weeks (mean \pm SD) } \\
\hline Positive symptom & $19.7 \pm 6.1$ & $16.6 \pm 6.2$ & $t=1.975$ & 0.053 \\
\hline Negative symptom & $16.0 \pm 6.5$ & $12.9 \pm 5.3$ & $t=2.071$ & 0.042 \\
\hline General psychopathology & $47.0 \pm 12.1$ & $37.6 \pm 11.8$ & $t=3.106$ & 0.003 \\
\hline \multicolumn{5}{|c|}{$\%$ Reduction in the severity of clinical symptoms (mean $\pm S D)^{e}$} \\
\hline Positive symptom & $36.6 \pm 16.4$ & $47.2 \pm 13.7$ & $t=-2.826$ & 0.006 \\
\hline Negative symptom & $48.3 \pm 15.7$ & $48.6 \pm 18.6$ & $t=-0.069$ & 0.946 \\
\hline General psychopathology & $28.0 \pm 11.2$ & $32.6 \pm 18.2$ & $t=-1.134$ & 0.261 \\
\hline
\end{tabular}

IM intermediate metabolizer, EM extensive metabolizer, SD standard deviation, ICV intracranial volume, APD antipsychotic drug, PANSS Positive and Negative Syndrome Scale.

${ }^{\mathrm{a}} \mathrm{APD}$ doses were converted to the equivalent of chlorpromazine.

' ${ }^{\text {Two }}$ subjects were taking lithium, and the others $(n=9)$ were taking divalproex.

cAntidepressants administered by subjects were escitalopram $(n=4)$, paroxetine $(n=2)$, and bupropion $(n=1)$.

$\mathrm{d}_{\%}$ reduction $\mathrm{In}$ the PANSS scores $=([$ Baseline-4-week follow-up $] /$ Baseline) $\times 100$.

'Levene's test for equality of variances indicated that the variances did not assumed to be equal between the two groups.

We further demonstrated that higher FAs and lower RDs, correlated with decreased CYP2D6 ASs, were associated with poor treatment response in positive symptoms in participants with schizophrenia. At first glance, this finding seems inconsistent with previous studies that suggested that decreased WM connectivity was a predictive marker for poor treatment outcome ${ }^{44,45}$. However, there are also alternative results showing that increased fronto-temporal WM connectivity at baseline was negatively associated with severity reduction in positive symptoms in patients with schizophrenia ${ }^{46,47}$. The discrepancy among these findings may partly be related to heterogeneity in study populations. A recent DTI study using a fully data-driven approach revealed distinct patterns of WM abnormalities in APD-naive, firstepisode patients with schizophrenia, suggesting neurobiological variability in the diagnostic category of schizophrenia ${ }^{48}$. Another possible speculation may involve a moderating role of CYP2D6 activity in the regulation of the central dopamine system and WM connectivity. As indicated by our findings, decreased activity of CYP2D6 seems to contribute to hyperactivity of the hippocampal dopamine system by enhancing hippocampal hyperactivity. This change could result in increased local redundant WM tracts ${ }^{49,50}$. Hence, higher FAs and lower RDs in IMs, and their lesser improvement in positive symptoms compared to EMs may indicate the additional dopaminergic burden $32,51,52$, owing to the low activity of CYP2D6 in the brain.

This study had several limitations to be considered when interpreting the results. First, other polymorphisms affecting CYP2D6 enzyme activity, such as $* 41$ or allele duplication, were not analyzed. However, since the frequencies of these polymorphisms are relatively low at $<5 \%$ of the total polymorphisms ${ }^{26}$, they would not change the main results. Second, although APD-naive or -free patients with schizophrenia were recruited at the enrollment, part of the MRI data was acquired after the initiation of APD treatment. Since the neurobiological effect of APDs on WM connectivity is very complex ${ }^{53,54}$, future studies will be warranted to exclude the confounding effect of APDs. Third, the direction of causality remains unknown owing to the cross-sectional design of this study. A prospective and longitudinal or long-term outcome approach is required to verify the causal direction in the association among CYP2D6 variation, WM connectivity, and the neurobiology of schizophrenia.

In conclusion, this was the first study to demonstrate the genetic influence of CYP2D6 polymorphism on hippocampal WM connectivity and the treatment response after APD treatment. Decreased CYP2D6 activity may confer susceptibility to the development of schizophrenia by increasing hippocampal hyperactivity and dopaminergic burden. Furthermore, CYP26Dassociated hippocampal WM alterations could be a possible endophenotype for schizophrenia that accounts for individual differences in clinical features and treatment response.

\section{METHODS \\ Participants}

Patients with schizophrenia were recruited from the Department of Psychiatry, CHA Bundang Medical Center (Seongnam, Republic of Korea). All participants were of Korean descent and met the diagnostic criteria for schizophrenia from the Diagnostic and Statistical Manual of Mental Disorders, Fourth Edition, Text Revision (DSM-IV-TR). The diagnostic interview was administered by the experienced psychiatrists using the Structured Clinical Interview for DSM-IV-TR Axis I Disorders ${ }^{55,56}$. We only recruited participants with schizophrenia who were APD-naive or free of APDs at least for 6 months. Participants were excluded if they had any current or past history of (1) other psychiatric disorders, including mood disorders and substance-related disorders; (2) intellectual disability; (3) medical or neurological disorders or head trauma with loss of consciousness; and (4) any other contraindications for MRI scan. Handedness was assessed using the Edinburgh Handedness Inventory ${ }^{57}$ and left-handed participants were excluded. A total of 106 participants with schizophrenia were finally included in the present study.

All study procedures were reviewed and approved by the Institutional Review Board of CHA Bundang Medical Center, in accordance with the latest version of the Declaration of Helsinki and principles of Good Clinical Practice. All participants provided written informed consent following a thorough explanation of the study procedures.

\section{Clinical assessment}

All participants with schizophrenia were assessed for the severity of their clinical symptoms using the PANSS ${ }^{30}$ at baseline. Sixty five participants 
(a)

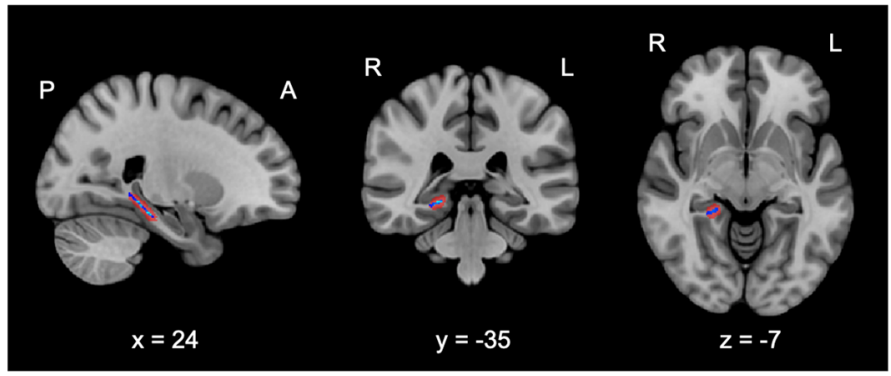

(c)

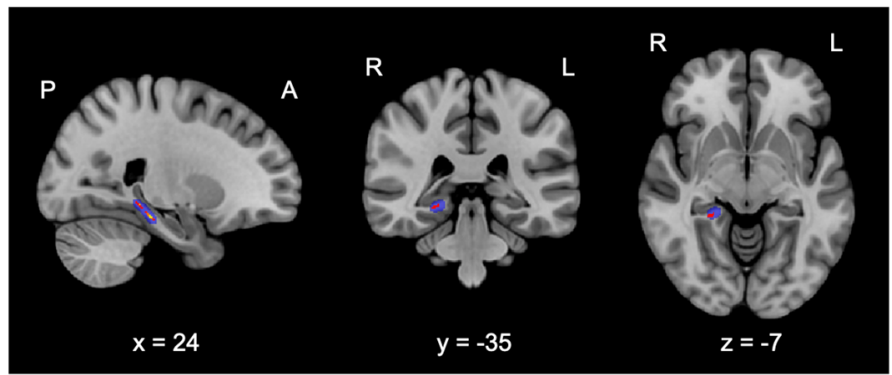

(b)

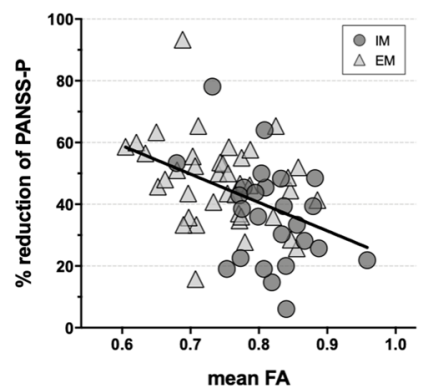

(d)

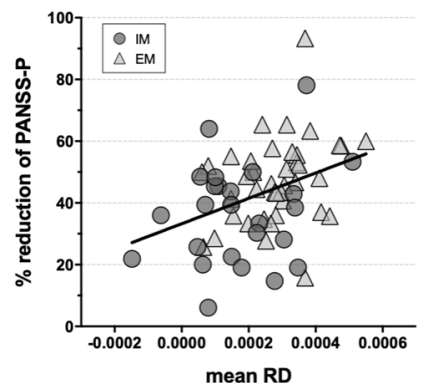

Fig. 3 Associations between CYP2D6-associated hippocampal white matter connectivity and treatment response after 4 weeks of APD treatment. Higher FAs $(\mathbf{a}, \mathbf{b})$ and lower RDs $(\mathbf{c}, \mathbf{d})$ were associated with lesser improvements in positive symptoms (TFCE-corrected $p<0.05$ ). The red (a) and blue areas (b) indicate the regions showing significant differences in FAs and RDs between the IM and EM groups, respectively. CYP2D6 cytochrome P450 2D6, APD antipsychotic drug, FA fractional anisotropy, RD radial diffusivity, PANSS-P the positive symptom subscale of the Positive and Negative Syndrome Scale, TFCE threshold-free cluster enhancement, IM intermediate metabolizer, EM extensive metabolizer.

(61.3\%) were followed-up with for the assessment of treatment response after 4 weeks of APD treatment as recommended in standard practice. Treatment response was defined as the percentage of reduction in each subscale of the PANSS (positive symptom, negative symptom, and general psychopathology) over 4 weeks. Since the PANSS adopts a 1-7 scoring system, the percentage of reduction in each subscale of the PANSS could not be $100 \%$, and it could be up to $85.7 \%$ (ref. ${ }^{58}$ ).

\section{Genotyping}

Genotyping was performed to analyze the CYP2D6*10 and CYP2D6*5 polymorphisms of the CYP2D6 gene. Briefly, the genomic DNA flanking the SNPs of interest was amplified using polymerase chain reaction (PCR) with forward and reverse primer pairs, and standard PCR reagents in $10 \mu \mathrm{L}$ reaction volume, containing $10 \mathrm{ng}$ of genomic DNA, $0.5 \mathrm{pM}$ of each oligonucleotide primer, $1 \mu \mathrm{L}$ of $10 \times$ PCR buffer, $250 \mu \mathrm{M}$ dNTP $(2.5 \mathrm{mM}$ each), and 0.25 units of i-StarTaq DNA polymerase ( 5 units $/ \mu \mathrm{L}$ ). The PCR reactions were performed as follows: 1 cycle at $95^{\circ} \mathrm{C}$ for $10 \mathrm{~min}$; followed by 35 cycles at $95^{\circ} \mathrm{C}$ for $30 \mathrm{~s}, 60^{\circ} \mathrm{C}$ for $1 \mathrm{~min}$, and $72^{\circ} \mathrm{C}$ for $1 \mathrm{~min}$; further followed by $1 \mathrm{cycle}$ at $72{ }^{\circ} \mathrm{C}$ for $10 \mathrm{~min}$. After amplification, the PCR products were treated with one unit each of shrimp alkaline phosphatase and exonuclease $\mathrm{I}$ at $37^{\circ} \mathrm{C}$ for $75 \mathrm{~min}$ and $72^{\circ} \mathrm{C}$ for $15 \mathrm{~min}$ to purify the amplified products. The $P C R$ products were sequenced using the $A B I$ PRISM SNaPShot Multiplex kit (Applied Biosystems; Foster City, CA, USA) and ABI PRISM 3730xI DNA analyzer (Applied Biosystems, Foster City; CA, USA). Primers of CYP2D6*10 used in this study were $5^{\prime}$-CATTGGTAGT GAGGCAGGT (forward primer), 5'-TGGTCGAAGCAGTATGGTG (reverse primer), and 5'-GCGCCAACGCTGGGCTGCACGCTAC (genotyping primer). The CYP2D6*5 allele was detected using the long PCR, as described previously ${ }^{59,60}$.

The CYP2D6 alleles were classified as the phenotypes of EM, IM, PM, and UM according to the expected enzyme activities reported on the database of the Pharmacogene Variation Consortium (PharmVar; https://www. pharmvar.org/gene/CYP2D6). Based on the Clinical Pharmacogenetics Implementation Consortium guidelines for CYP2D6 genotype and codeine therapy ${ }^{61}, \mathrm{AS}$ values were assigned to each allele as follows: 0 for PM; 0.5-1.0 for IM; 1.5-2.0 for EM; and >2.0 for UM. The total CYP2D6 AS was calculated as the sum of the values assigned to each allele (CYP2D $* 5=0$, CYP2D6* $10(T)=0.5, C Y P 2 D 6 * 10(C)=1.0$ [major allele: $C$, minor allele: $T]$ ).

\section{MRI data acquisition}

MRI data acquisition was performed at CHA Bundang Medical Center using a 3.0-Tesla GE Signa HDxt scanner (GE Healthcare, Milwaukee, WI, USA). Diffusion-weighted images (DWI) were acquired using an echo planar imaging (EPI) sequence with the following parameters: repetition time, $17,000 \mathrm{~ms}$; echo time, $108 \mathrm{~ms}$; field of view, $240 \mathrm{~mm}$; matrix, $144 \times 144$; slice thickness, $1.7 \mathrm{~mm}$; and voxel size, $1.67 \times 1.67 \times 1.7 \mathrm{~mm}^{3}$. A doubleecho option was applied to reduce eddy-current-related distortions. To reduce the impact of EPI spatial distortions, an 8-channel coil and ASSET (Array of Spatial Sensitivity Encoding Techniques; GE Healthcare) with a SENSE factor of two was used. Seventy axial slices parallel to the anterior commissure-posterior commissure line were acquired in 51 directions with $b=900 \mathrm{~s} / \mathrm{mm}^{2}$. Eight baseline scans with $b=0 \mathrm{~s} / \mathrm{mm}^{2}$ were also acquired. DTls were extracted from the DWls using the least-squares method.

\section{DTI analysis}

Analysis of DTI data was conducted using the Functional MRI of the Brain (FMRIB) Diffusion Toolbox and Tract-based Spatial Statistics (TBSS), implemented in the FMRIB Software Library (FSL version 6.0; Oxford, UK; https://fsl.fmrib.ox.ac.uk/fsl/). DTls underwent standard preprocessing steps, including skull stripping and eddy-current correction. FA images were created by fitting a tensor model to the corrected diffusion data and then aligned in the Montreal Neurologic Institute standard space. Transformed FA images were combined and applied to the original FA map to produce a standard-space version of the FA map. A mean FA image, created by averaging all transformed FA images, was thinned to obtain a mean FA skeleton representing the centers of the WM tracts. The skeleton was thresholded using an FA $>0.2$ to involve only major fiber bundles. Non-FA (MD, AD, and $R D$ ) images were prepared in a similar way as provided by TBSS.

Prior to conducting voxel-wise statistical analysis of DTI data, hippocampal WM regions ${ }^{62,63}$ (hippocampal cingulum, fornix, and stria terminalis) were chosen as ROIs based on the JHU DTI-based probabilistic tractography atlas ${ }^{29}$. Statistical analysis of DTI data was performed within the ROI mask using permutation-based nonparametric inference within the framework of a general linear model. There were 10000 permutations, and multiple comparisons were adjusted using the threshold-free cluster enhancement (TFCE) method. Analysis of covariance with sex, age, ICV, duration of illness, and APD dose at scan as covariates was additionally 
conducted to rule the confounding effects of these variables on the results. Significance was set at $p<0.05$.

\section{Statistical analyses}

To compare the sociodemographic and clinical characteristics, independent $t$ tests for continuous variables and chi-squared tests for categorical variables were used. Correlation analyses were performed to confirm the associations among CYP2D6 activity, hippocampal WM connectivity, and treatment response. Statistical procedures were performed using SPSS version 26 (IBM Corporation; Armonk, NY, USA). Significance was set at $p<$ 0.05 .

\section{Reporting summary}

Further information on research design is available in the Nature Research Reporting Summary linked to this article.

\section{DATA AVAILABILITY}

The data supporting the findings of this study are not publicly available due to ethical restrictions for protecting participants' confidentiality and privacy, but are accessible from the corresponding author on reasonable request with the approval of the Institutional Review Board of CHA Bundang Medical Center.

\section{CODE AVAILABILITY}

No custom code or mathematical algorithm was used in this study.

Received: 12 July 2020; Accepted: 12 November 2020; Published online: 29 January 2021

\section{REFERENCES}

1. Gaedigk, A. et al. The CYP2D6 activity score: translating genotype information into a qualitative measure of phenotype. Clin. Pharm. Ther. 83, 234-242 (2008).

2. Chinta, S. J. et al. Constitutive expression and localization of the major drug metabolizing enzyme, cytochrome P4502D in human brain. Brain Res. Mol. Brain Res. 103, 49-61 (2002).

3. Siegle, I. et al. Cellular localization and regional distribution of CYP2D6 mRNA and protein expression in human brain. Pharmacogenetics 11, 237-245 (2001).

4. Funae, Y. et al. CYP2D in the brain. Drug Metab. Pharmacokinet. 18, 337-349 (2003).

5. Ozdemir, V. et al. CYP2D6 genotype in relation to perphenazine concentration and pituitary pharmacodynamic tissue sensitivity in Asians: CYP2D6-serotonindopamine crosstalk revisited. Pharmacogenet. Genomics 17, 339-347 (2007).

6. Britto, M. R. \& Wedlund, P. J. Cytochrome P-450 in the brain. Potential evolutionary and therapeutic relevance of localization of drug-metabolizing enzymes. Drug Metab. Dispos. 20, 446-450 (1992).

7. Andersen, P. H. Biochemical and pharmacological characterization of $[3 \mathrm{H}] \mathrm{GBR}$ 12935 binding in vitro to rat striatal membranes: labeling of the dopamine uptake complex. J. Neurochem. 48, 1887-1896 (1987).

8. Niznik, H. B. et al. The dopamine transporter and cytochrome P45OIID1 (debrisoquine 4-hydroxylase) in brain: resolution and identification of two distinct [3H] GBR-12935 binding proteins. Arch. Biochem. Biophys. 276, 424-432 (1990).

9. Hiroi, T. et al. Specific binding of 1-[2-(diphenylmethoxy)ethyl]-4-(3-phenyl propyl) piperazine (GBR-12935), an inhibitor of the dopamine transporter, to human CYP2D6. Biochem. Pharmacol. 53, 1937-1939 (1997).

10. Hiroi, T., Imaoka, S. \& Funae, Y. Dopamine formation from tyramine by CYP2D6. Biochem. Biophys. Res. Commun. 249, 838-843 (1998).

11. McCann, S. J., Pond, S. M., James, K. M. \& Le Couteur, D. G. The association between polymorphisms in the cytochrome P-450 2D6 gene and Parkinson's disease: a case-control study and meta-analysis. J. Neurol. Sci. 153, 50-53 (1997).

12. Saarikoski, S. T. et al. CYP2D6 ultrarapid metabolizer genotype as a potential modifier of smoking behaviour. Pharmacogenetics 10, 5-10 (2000).

13. Gonzalez, I. et al. Relation between CYP2D6 phenotype and genotype and personality in healthy volunteers. Pharmacogenomics 9, 833-840 (2008).

14. Zackrisson, A. L., Lindblom, B. \& Ahlner, J. High frequency of occurrence of CYP2D6 gene duplication/multiduplication indicating ultrarapid metabolism among suicide cases. Clin. Pharm. Ther. 88, 354-359 (2010).

15. Daniels, J. et al. No association between schizophrenia and polymorphisms within the genes for debrisoquine 4-hydroxylase (CYP2D6) and the dopamine transporter (DAT). Am. J. Med. Genet. 60, 85-87 (1995).
16. Jonsson, E. G. et al. Lack of association between debrisoquine 4-hydroxylase (CYP2D6) gene polymorphisms and schizophrenia. Psychiatr. Genet. 8, 25-28 (1998).

17. Chen, C. H., Hung, C. C., Wei, F. C. \& Koong, F. J. Debrisoquine 4-hydroxylase (CYP2D6) genetic polymorphisms and susceptibility to schizophrenia in Chinese patients from Taiwan. Psychiatr. Genet. 11, 153-155 (2001).

18. Llerena, A. et al. Low frequency of CYP2D6 poor metabolizers among schizophrenia patients. Pharmacogenomics J. 7, 408-410 (2007).

19. Grace, A. A. Dysregulation of the dopamine system in the pathophysiology of schizophrenia and depression. Nat. Rev. Neurosci. 17, 524-532 (2016).

20. Kapur, S. Psychosis as a state of aberrant salience: a framework linking biology, phenomenology, and pharmacology in schizophrenia. Am. J. Psychiatry 160, 13-23 (2003).

21. Cai, W. M., Chen, B. \& Zhang, W. X. Frequency of CYP2D6*10 and *14 alleles and their influence on the metabolic activity of CYP2D6 in a healthy Chinese population. Clin. Pharm. Ther. 81, 95-98 (2007).

22. Thomason, M. E. et al. COMT genotype affects prefrontal white matter pathways in children and adolescents. Neuroimage 53, 926-934 (2010).

23. Seeman, P. Dopamine D2 receptors as treatment targets in schizophrenia. Clin. Schizophr. Relat. Psychoses 4, 56-73 (2010).

24. Ingelman-Sundberg, M., Sim, S. C., Gomez, A. \& Rodriguez-Antona, C. Influence of cytochrome P450 polymorphisms on drug therapies: pharmacogenetic, pharmacoepigenetic and clinical aspects. Pharm. Ther. 116, 496-526 (2007).

25. Gasche, Y. et al. Codeine intoxication associated with ultrarapid CYP2D6 metabolism. N. Engl. J. Med. 351, 2827-2831 (2004).

26. Lee, S. Y. et al. Sequence-based CYP2D6 genotyping in the Korean population. Ther. Drug Monit. 28, 382-387 (2006).

27. Roh, H. K. et al. Debrisoquine and S-mephenytoin hydroxylation phenotypes and genotypes in a Korean population. Pharmacogenetics 6, 441-447 (1996).

28. Sohn, D. R. et al. Metoprolol oxidation polymorphism in a Korean population: comparison with native Japanese and Chinese populations. Br. J. Clin. Pharm. 32, 504-507 (1991).

29. Mori, S. \& Aggarwal, M. In vivo magnetic resonance imaging of the human limbic white matter. Front Aging Neurosci. 6, 321 (2014).

30. Kay, S. R., Fiszbein, A. \& Opler, L. A. The positive and negative syndrome scale (PANSS) for schizophrenia. Schizophr. Bull. 13, 261-276 (1987).

31. Kirchheiner, J. et al. CYP2D6 in the brain: genotype effects on resting brain perfusion. Mol. Psychiatry 16, 333-241 (2011).

32. Medoff, D. R., Holcomb, H. H., Lahti, A. C. \& Tamminga, C. A. Probing the human hippocampus using rCBF: contrasts in schizophrenia. Hippocampus 11, 543-550 (2001).

33. Malaspina, D. et al. Resting neural activity distinguishes subgroups of schizophrenia patients. Biol. Psychiatry 56, 931-937 (2004).

34. Kawasaki, Y. et al. Regional cerebral blood flow in patients with schizophrenia. A preliminary report. Eur. Arch. Psychiatry Clin. Neurosci. 241, 195-200 (1992).

35. Schobel, S. A. et al. Imaging patients with psychosis and a mouse model establishes a spreading pattern of hippocampal dysfunction and implicates glutamate as a driver. Neuron 78, 81-93 (2013).

36. Heckers, S. \& Konradi, C. GABAergic mechanisms of hippocampal hyperactivity in schizophrenia. Schizophr. Res. 167, 4-11 (2015).

37. Grace, A. A. Dopamine system dysregulation by the hippocampus: implications for the pathophysiology and treatment of schizophrenia. Neuropharmacology 62, 1342-1348 (2012)

38. Stadelmann, C., Timmler, S., Barrantes-Freer, A. \& Simons, M. Myelin in the central nervous system: structure, function, and pathology. Physiol. Rev. 99, 1381-1431 (2019).

39. Giegling, I. et al. Interaction of haloperidol plasma level and antipsychotic effect in early phases of acute psychosis treatment. J. Psychiatr. Res. 44, 487-492 (2010).

40. Medori, R., Mannaert, E. \& Grunder, G. Plasma antipsychotic concentration and receptor occupancy, with special focus on risperidone long-acting injectable. Eur. Neuropsychopharmacol. 16, 233-240 (2006).

41. Perry, P. J., Sanger, T. \& Beasley, C. Olanzapine plasma concentrations and clinical response in acutely ill schizophrenic patients. J. Clin. Psychopharmacol. 17, 472-477 (1997).

42. Rosenzweig, P. et al. A review of the pharmacokinetics, tolerability and pharmacodynamics of amisulpride in healthy volunteers. Hum. Psychopharmacol. 17, 1-13 (2002)

43. de Leon, J., Wynn, G. \& Sandson, N. B. The pharmacokinetics of paliperidone versus risperidone. Psychosomatics 51, 80-88 (2010).

44. Kraguljac, N. V. et al. White matter integrity, duration of untreated psychosis, and antipsychotic treatment response in medication-naive first-episode psychosis patients. Mol. Psychiatry https://doi.org/10.1038/s41380-020-0765-x (2020).

45. Reis Marques, T. et al. White matter integrity as a predictor of response to treatment in first episode psychosis. Brain 137, 172-182 (2014). 
46. Cho, S. J. et al. White matter integrity associated with severity reductions in positive symptoms after amisulpride treatment in drug-free patients with schizophrenia. Neurosci. Lett. 685, 131-136 (2018).

47. Kim, M. K. et al. White-matter connectivity related to paliperidone treatment response in patients with schizophrenia. J. Psychopharmacol. 30, 294-302 (2016).

48. Sun, $\mathrm{H}$. et al. Two patterns of white matter abnormalities in medication-naive patients with first-episode schizophrenia revealed by diffusion tensor imaging and cluster analysis. JAMA Psychiatry 72, 678-686 (2015).

49. Canu, E., Agosta, F. \& Filippi, M. A selective review of structural connectivity abnormalities of schizophrenic patients at different stages of the disease. Schizophr. Res. 161, 19-28 (2015).

50. Guillin, O., Abi-Dargham, A. \& Laruelle, M. Neurobiology of dopamine in schizophrenia. Int. Rev. Neurobiol. 78, 1-39 (2007).

51. Tregellas, J. R. et al. Intrinsic hippocampal activity as a biomarker for cognition and symptoms in schizophrenia. Am. J. Psychiatry 171, 549-556 (2014).

52. Lahti, A. C. et al. Modulation of limbic circuitry predicts treatment response to antipsychotic medication: a functional imaging study in schizophrenia. Neuropsychopharmacology 34, 2675-2690 (2009).

53. Zeng, B. et al. Abnormal white matter microstructure in drug-naive first episode schizophrenia patients before and after eight weeks of antipsychotic treatment. Schizophr. Res. 172, 1-8 (2016).

54. Szeszko, P. R. et al. White matter changes associated with antipsychotic treatment in first-episode psychosis. Neuropsychopharmacology 39, 1324-1331 (2014).

55. Farmer, R. F. \& Chapman, A. L. Evaluation of DSM-IV personality disorder criteria as assessed by the structured clinical interview for DSM-IV personality disorders. Compr. Psychiatry 43, 285-300 (2002).

56. First, M., Spitzer, R., Gibbon, M. \& Williams, J. Structured Clinical Interview for DSMIV Axis I Disorders (SCID) (New York State Psychiatric Institute, New York 1995).

57. Oldfield, R. C. The assessment and analysis of handedness: the Edinburgh inventory. Neuropsychologia 9, 97-113 (1971).

58. Leucht, S. et al. Defining 'response' in antipsychotic drug trials: recommendations for the use of scale-derived cutoffs. Neuropsychopharmacology 32, 1903-1910 (2007).

59. Hersberger, M., Marti-Jaun, J., Rentsch, K. \& Hanseler, E. Rapid detection of the CYP2D6*3, CYP2D6*4, and CYP2D6*6 alleles by tetra-primer PCR and of the CYP2D6*5 allele by multiplex long PCR. Clin. Chem. 46, 1072-1077 (2000).

60. Ishiguro, A., Kubota, T., Sasaki, H. \& Iga, T. A long PCR assay to distinguish CYP2D6*5 and a novel CYP2D6 mutant allele associated with an 11-kb EcoRI haplotype. Clin. Chim. Acta 347, 217-221 (2004).

61. Crews, K. R. et al. Clinical Pharmacogenetics Implementation Consortium guidelines for cytochrome P450 2D6 genotype and codeine therapy: 2014 update. Clin. Pharm. Ther. 95, 376-382 (2014).

62. Hoogenboom, W. S. et al. Limbic system white matter microstructure and longterm treatment outcome in major depressive disorder: a diffusion tensor imaging study using legacy data. World J. Biol. Psychiatry 15, 122-134 (2014).

63. Concha, L., Gross, D. W. \& Beaulieu, C. Diffusion tensor tractography of the limbic system. Am. J. Neuroradiol. 26, 2267-2274 (2005).

\section{ACKNOWLEDGEMENTS}

This research was supported by the Basic Science Research Program (grant no. NRF2018R1D1A1B07050493 to D.-Y.C.) and Bio and Medical Technology Development
Program (grant nos. NRF-2016M3A9E8941670 to M.B. and NRF-2019M3C7A1032262 to S.-H.L.) of the National Research Foundation funded by the Ministry of Science and ICT, Republic of Korea. The funding sources had no role in study design; in the collection, analysis, and interpretation of data; in the writing of the report; or in the decision to submit the paper for publication.

\section{AUTHOR CONTRIBUTIONS}

W.S., D.-Y.C., and S.-H.L. designed the study. S.-H.L. and M.B. managed participant recruitment and data acquisition, compiled the database, and conducted data preprocessing. W.S. and A.K. undertook statistical analysis. W.S., A.K., and M.B. implemented the literature reviews and data interpretation. W.S. wrote the first draft of the manuscript, and M.B. revised the analyses and provided critical revisions. S.-H.L. supervised the manuscript. All authors contributed to and approved the final manuscript.

\section{COMPETING INTERESTS}

The authors declare no competing interests.

\section{ADDITIONAL INFORMATION}

Supplementary information is available for this paper at https://doi.org/10.1038/ s41537-020-00134-z.

Correspondence and requests for materials should be addressed to S.-H.L.

Reprints and permission information is available at http://www.nature.com/ reprints

Publisher's note Springer Nature remains neutral with regard to jurisdictional claims in published maps and institutional affiliations.

\begin{abstract}
Open Access This article is licensed under a Creative Commons Attribution 4.0 International License, which permits use, sharing, daptation, distribution and reproduction in any medium or format, as long as you give appropriate credit to the original author(s) and the source, provide a link to the Creative Commons license, and indicate if changes were made. The images or other third party material in this article are included in the article's Creative Commons license, unless indicated otherwise in a credit line to the material. If material is not included in the article's Creative Commons license and your intended use is not permitted by statutory regulation or exceeds the permitted use, you will need to obtain permission directly from the copyright holder. To view a copy of this license, visit http://creativecommons. org/licenses/by/4.0/.
\end{abstract}

(c) The Author(s) 2021 